\title{
Um inimigo invisível: COVID-19 e o seu impacto à saúde mental
}

\author{
An invisible enemy: COVID-19 and its impact on mental health \\ Un enemigo invisible: COVID-19 y su impacto en la salud mental
}

Recebido: 14/08/2021 | Revisado: 19/08/2021 | Aceito: 20/08/2021 | Publicado: 22/08/2021

\author{
Raíssa Ester Maia Monteiro \\ ORCID: https://orcid.org/0000-0001-8558-2043 \\ Universidade Federal da Paraíba, Brasil \\ E-mail: raissaestermm@gmail.com \\ Cynthia Borges Luiz \\ ORCID: https://orcid.org/0000-0001-8409-6617 \\ Centro Universitário de João Pessoa, Brasil \\ E-mail: cynthiab12018@gmail.com \\ Emerson Serafim Galdino Monteiro \\ ORCID: https://orcid.org/ 0000-0002-1057-4281 \\ Médico Psiquiatra, Brasil \\ E-mail: emersonsgmonteiro@gmail.com \\ Luiz Carlos Serramo Lopez \\ ORCID: https://orcid.org/0000-0002-9983-5563 \\ Universidade Federal da Paraíba, Brasil \\ E-mail: lcslopez@gmail.com
}

\begin{abstract}
Resumo
O COVID-19 trouxe diversos desafios para o Brasil e para o mundo. Além dos impactos biológicos, sociais e econômicos, o novo coronavírus vem comprometendo sobremaneira a saúde mental das pessoas, seja pela possibilidade de exposição ao contágio, pelo impacto advindo do distanciamento social ou pelo medo da morte. Esse estudo se trata de uma revisão narrativa de literatura mediante o uso da pesquisa bibliográfica, de pesquisa descritiva e natureza qualitativa, com o objetivo de apresentar conhecimentos relativos à saúde mental durante o COVID-19, especificamente sobre os impactos à saúde mental ocasionados pelo vírus. Buscou-se por materiais em diversas bases de dados (Google Scholar, SciELO, PubMed, Lilacs, entre outros), bem como se utilizou de alguns descritores para filtrar a busca pelo conteúdo, a exemplo de "Covid-19", "Sars-Cov-2", "pandemia", "distanciamento social" e "saúde mental". À guisa de conclusão, a literatura indica que boa parte da população foi afetada, desenvolvendo algum transtorno mental nesse período, com destaque para a ansiedade e a depressão. Importa a criação de estratégias para promover qualidade de vida e bem-estar em tempos tão propícios para o adoecimento mental.
\end{abstract}

Palavras-chave: COVID-19; Pandemia; Saúde mental.

\begin{abstract}
COVID-19 brought several challenges to Brazil and the world. In addition to the biological, social and economic impacts, the new coronavirus has greatly compromised people's mental health, whether due to the possibility of exposure to contagion, the impact arising from social distancing or the fear of death. This study is a narrative literature review using bibliographical research, descriptive research and qualitative nature, with the aim of presenting knowledge related to mental health during COVID-19 specifically on the impacts on mental health caused by the virus. We searched for materials in several databases (Google Scholar, SciELO, PubMed, Lilacs, among others), as well as using some descriptors to filter the search by content, such as "Covid-19", "Sars- Cov-2", "pandemic", "social distancing" and "mental health". In conclusion, the literature indicates that a good part of the population was affected, developing some mental disorder during this period, especially anxiety and depression. It is important to create strategies to promote quality of life and well-being in times that are so favorable for mental illness.
\end{abstract}

Keywords: COVID-19; Pandemic; Mental health.

\section{Resumen}

COVID-19 trajo varios desafíos a Brasil y al mundo. Además de los impactos biológicos, sociales y económicos, el nuevo coronavirus ha comprometido enormemente la salud mental de las personas, ya sea por la posibilidad de exposición al contagio, el impacto derivado del distanciamiento social o el miedo a la muerte. Este estudio es una revisión de literatura narrativa utilizando investigación bibliográfica, investigación descriptiva y de carácter cualitativo, con el objetivo de presentar conocimientos relacionados con la salud mental durante el COVID-19 específicamente sobre los impactos en la salud mental causados por el virus. Se realizaron búsquedas de materiales en varias bases de datos (Google Scholar, SciELO, PubMed, Lilacs, entre otras), además de utilizar algunos descriptores para filtrar la búsqueda por contenido, como "Covid-19", "Sars-Cov-2", "Pandemia", "distanciamiento social" y "salud mental". En conclusión, la literatura indica que buena parte de la población se vio afectada, desarrollando 
algún trastorno mental durante este período, especialmente ansiedad y depresión. Es importante crear estrategias para promover la calidad de vida y el bienestar en momentos tan favorables para la enfermedad mental.

Palabras clave: COVID-19; Pandemia; Salud mental.

\section{Introdução}

A potencialidade da ação do COVID-19 foi e está sendo bastante abrupta. Seu poder é tão ferrenho que mesmo após pouco mais de um ano de sua visibilidade, a nível mundial, permanece gerando muitas mudanças em diversos setores da sociedade, seja a nível político, social, econômico, mudanças repentinas de estilos de vida e relações familiares e sociais.

Seu impacto no cenário social, político e econômico é evidente, uma vez que arraigado à pandemia pode-se também identificar um arcabouço de processos e rupturas que compõem um fenômeno recheado de aspectos que giram em torno da dor, sofrimento, medo, insegurança, ansiedade, depressão, os quais vêm afetando a saúde mental das pessoas e marcando toda uma era.

A pandemia evidencia uma situação de emergência extrema, ao mesmo tempo em que explicita nossas qualidades e defeitos como seres humanos. Desde então, o SARS-CoV-2 disseminou-se por todos os continentes levando à óbito milhões de pessoas ao redor do mundo. O desafio de conter essa pandemia, de significativa magnitude, tem sido constante, principalmente aos países de média e baixa renda, a exemplo do Brasil, que carece da tecnologia necessária ficando, dessa forma, à mercê de outros países (Iser et al., 2020).

Além dos inúmeros impactos, sejam eles biológicos, sociais e econômicos, o novo coronavírus vem impactando sobremaneira a saúde mental das pessoas, seja pela insegurança decorrente da exposição ao contágio, pelo impacto advindo do distanciamento social, pelo medo da morte ou por traumas de quem já adoeceu pelo vírus e precisaram de cuidados médicos mais intensos.

Destarte, os efeitos psicossociais ocasionados pela pandemia do COVID-19 levam a crê na existência de uma "pandemia do medo e estresse", especialmente em virtude da ausência de controle eficaz e mecanismos terapêuticos suficientes que geram insegurança e incertezas à população.

Sendo assim, o presente estudo tem por objetivo elucidar os impactos da pandemia por COVID-19 na saúde mental que vêm sendo apontados pela literatura, para que assim possam ser desenvolvidas estratégias de promoção de saúde e tratamento para o adoecimento mental, visando o bem-estar e qualidade de vida da sociedade.

\section{Metodologia}

Trata-se de um estudo qualitativo de revisão narrativa de literatura, a qual permitiu a reunião e exame de trabalhos com abordagens metodológicas diversas, de literatura empírica e teórica ou, nas palavras de Laville \& Dionne (1999), objetivando selecionar e refletir aquilo que foi relevante para a presente pesquisa com uma apresentação descritiva dos achados, de modo que os trabalhos disponíveis sejam revisados e selecionando-se tudo que seja útil à pesquisa.

Assim, através de uma pesquisa bibliográfica ou de fonte secundária, que utiliza "levantamento de referências já publicadas, em forma de artigos científicos (impressos ou virtuais), livros, teses de doutorado, dissertações de mestrado" (Marconi \& Lakatos, 2017, p. 320) buscou-se por materiais em diversas bases de dados (Google Scholar, SciELO, PubMed, Lilacs, entre outros), assim como sites de organizações relacionadas ao COVID-19 e o mundo trabalho (OMS, OIT, Ministério da Saúde, etc). Utilizou-se da escolha de alguns descritores para filtrar a busca pelo conteúdo, a exemplo de "Covid-19”, "SarsCov-2" e "saúde mental". As informações foram sintetizadas, no período de março a agosto de 2021, a partir de uma gama variada de estudos, sejam eles artigos, dissertações, teses, livros, bem como acesso a sites de organizações intrinsecamente relacionadas com a temática em questão, tais como o site da OMS (Organização Mundial da Saúde) e Ministério da Saúde. 
De modo a aperfeiçoar a busca, optou-se por limitar quanto ao quesito tempo estudos datados entre 2019 a 2021, período em que despontou de forma mais abrupta questões referentes à temática em referência. Os achados da presente revisão narrativa de literatura estão dispostos em 2 seções intituladas respectivamente: "Breves reflexões sobre o Covid-19" e a "Relação entre Covid-19 e saúde mental".

\section{Resultados e Discussão}

\subsection{Breves reflexões sobre o Covid-19}

A doença COVID-19 (Coronavirus Disease 2019), ocasionada pelo Coronavírus da Síndrome Respiratória Aguda Grave 2 (SARS-CoV-2), trouxe diversos impactos para o Brasil e para o mundo. A saúde pública, a nível internacional, ficou em alerta especialmente após a constatação da rápida disseminação do vírus. Dessa maneira, a World Health Organization (WHO) caracterizou o COVID-19 uma pandemia, no dia 11 de março de 2020 (BVS, 2021).

A pandemia do COVID-19, tendo como a síndrome gripal (SG) a manifestação mais comum, teve sua notoriedade a partir do final do ano de 2019, em Wuhan, Província de Hubei, China, em virtude de um surto de pneumonia de origem desconhecida, sendo até então concebida como uma epidemia (Sifuentes-Rodríguez \& Palacios-Reyes, 2020).

Estimam-se 121.969.223 de casos confirmados e 2.694.094 de mortes confirmadas até a data de 21 de março de 2021. A título de atualização, em 13 de agosto de 2021 segundo a World Health Organization, são 205.338 .159 de casos conformados e 4.333.094 de mortes confirmadas (WHO, 2021). No Brasil, o primeiro caso notificado da doença ocorreu em 25 de fevereiro de 2020, sendo o número de casos crescente desde então, ocasionando inúmeras consequências a nível econômico, político e social (Duarte et al., 2020).

O primeiro óbito, no Brasil, em decorrência do COVID-19 ocorreu em 17 de março de 2020, homem idoso, que apresentava diabetes e hipertensão, não sendo constatada viagem recente ao exterior. No dia 20 de março do mesmo ano houve o reconhecimento de contaminação comunitária em todo o Brasil (Oliveira et al., 2020; Cavalcante et al., 2020).

Em números absolutos, a região Sudeste concentrou até então a maior quantidade de casos, embora a região Norte tenha indicado a maior incidência do COVID-19 até o momento da Semana Epidemiológica (SE) 20 de 2020. Ressalta-se, ainda, que nessa região, foi registrado um dos maiores índices de falta de adesão ao distanciamento social por parte da população. Ademais, a região Norte apresenta uma rede hospitalar menor quando comparada à rede hospitalar de outras regiões. Logo, a quantidade de leitos é pequena quando comparada à demanda, seja em rede pública ou privada. Assim, apresentou o maior número de mortalidade desde a 18 $8^{\mathrm{a}} \mathrm{SE}$ de 2020 (Cavalcante et al., 2020).

Segundo boletim epidemiológico divulgado pelo Ministério da Saúde referente à SE 8 de 2021 (21 a 27/02/2021), em 04 de março de 2021, desde as SE's de 2020 até a SE 8 de 2021 os casos confirmados e óbitos decorrentes do COVID-19 se apresentaram bastante heterogêneos entre as regiões brasileiras. Na SE 8, o Sudeste apresentava o número mais significativo de casos novos, seguido do Sul, Nordeste, Centro-Oeste e Norte. No que diz respeito aos óbitos, o Sudeste também liderou o número absoluto no país, seguido pela Região Nordeste, Sul, Norte e Centro-Oeste do Brasil (Brasil, 2021a).

Com base nos dados diários informados pelas SES ao MS e segundo informações obtidas através do site do Ministério da Saúde, o Brasil registra, de 26 de fevereiro de 2020 a 7 de agosto de 2021 (semana epidemiológica n ${ }^{\circ}$ 31), a confirmação de 20.151.779 casos e 562.752 óbitos por covid-19 no Brasil. Ao final da SE 31 foram 18.894.631 milhões de pessoas recuperadas. Outros 694.396 são casos que continuam em acompanhamento (Brasil, 2021b).

Desde a declaração emitida pela World Health Organization do enfrentamento de um surto de novo coronavírus (SARS-CoV-2), com grande alarme a nível internacional, a nível de pandemia, houve uma grande investida por parte dos governos e laboratórios para a criação de vacinas contra o COVID-19 (Domingues, 2021). 
Segundo o Plano Nacional de Operacionalização da Vacinação contra a COVID-19, em sua $4^{\mathrm{a}}$ edição, em fevereiro de 2021, diante da situação emergencial e necessidade de se disponibilizar as vacinas, a agência reguladora ANVISA autorizou, em caráter emergencial e experimental, dois processos referentes à vacina do COVID-19, submetidos à agência: Instituto Butantan (IB) Coronavac, cuja fabricante é a Sinovac Life Sciences Co., Ltd. Parceria: IB/ Sinovac e da Fundação Oswaldo Cruz (Fiocruz), cuja fabricante é a Serum Institute of India Pvt. Ltd. Parceria: Fiocruz/ Astrazeneca (Brasil, 2021 c).

Diversos estudos foram realizados a fim de apontar sinais e sintomas relacionados ao COVID-19, a exemplo dos estudos de Guan et al. (2020); Wang et al. (2020) \& Mao et al. (2020), os quais apontam que a sintomatologia mais comumente apontada seria a febre $\left(\geq 37,8^{\circ} \mathrm{C}\right)$, tosse, dispneia (dificuldade respiratória), mialgia e fadiga. Devido à heterogeneidade das apresentações clínicas, o diagnóstico ainda não é tão simples, embora aparentemente haja um relativo consenso de que a população com idade avançada ou que apresente comorbidades estejam mais vulneráveis às piores evoluções (Iser et al., 2020).

O COVID-19 se trata de uma infecção viral de alto poder de transmissão. Presume-se que esse vírus é transmitido por gotículas advindas de tosse, espirro ou até mesmo a fala de uma pessoa contaminada, secreções, contato com a boca ou nariz. É válido ressaltar que a transmissão via oral-fecal (vírus presente nas fezes de uma pessoa infectada, com o poder de contaminar alimentos ou a água) é uma situação que ainda não foi descartada (Pereira et al., 2020).

Uma importante estratégia utilizada pelo Ministério da Saúde (MS) desde o início foi a informação e comunicação, seja através de boletins epidemiológicos frequentes, uso da imprensa, entrevistas coletivas diárias, disponibilização de novos meios de atendimento à população, a exemplo do aplicativos "Coronavírus- SUS". O MS vem atuando no reforço à assistência da saúde da população com ações de contratação e capacitação de profissionais da saúde, especialmente profissionais médicos. Para que a assistência seja oferecida de forma segura, o MS também investiu na distribuição de equipamentos de proteção individual (EPI), construção de novos hospitais e ampliação dos já existentes (Oliveira et al, 2020).

No que tange à procura e disponibilidade de vagas em hospitais e equipamentos que auxiliem a respiração do paciente, a exemplo da ventilação assistida, ressalta-se a urgência da necessidade de desacelerar a propagação da COVID-19 para que se tenha tempo necessário e suficiente para oferecer um serviço de qualidade nos diversos níveis de prestação de atendimento à saúde. Ressalta-se, oportunamente, que a colaboração e parceria dos hospitais privados para o amortecimento do déficit de demanda e a necessidade de reorganização dos serviços de saúde, especialmente quanto à regionalização, são de suma importância. Isso porque, durante tempos de pandemia, o desenho de regionalização apresenta muitos entraves para o alcance de certas populações, em especial na Região Norte (Noronha et al., 2020).

Indubitavelmente, a pandemia gerada pelo vírus Sars-CoV-2 trouxe novas regras e hábitos para toda a população mundial, a qual se viu diante da necessidade de adotar recomendações, estratégias e medidas de prevenção. No Brasil, medidas de controle e prevenção vem sendo tomadas e adotadas, a nível de governo federal, estadual e municipal, sendo a medida do distanciamento social a mais difundida pelas autoridades, embora também tenha sido a mais polêmica, quando as pessoas foram orientadas a permanecerem em seus domicílios, saindo das suas casas tão somente em casos de extrema necessidade, para que a disseminação do vírus fosse diminuída (Bezerra et al., 2020).

Pela alta taxa de pessoas assintomáticas, o distanciamento social reduziu, mas não resolveu o problema do avanço do vírus, sendo constatado ainda nos dias atuais, números significativos de óbitos em decorrência do COVID-19 em todo o mundo. Além da ferramenta do distanciamento social, outras medidas profiláticas são recomendadas para reduzir a proliferação do vírus, a nível individual (Malta et al., 2020).

Entre as principais medidas, é possível citar a título de exemplo, o uso de máscaras, o não compartilhamento de objetos pessoais, lavar as mãos com sabão ou álcool a 70\%, substituir a máscara quando a mesma estiver úmida por uma máscara nova; a nível ambiental, a limpeza cuidadosa e rotineira dos ambientes e superfícies, bem como a manutenção de 
locais arejados e ventilados; e comunitário, como a inclusão de algumas medidas de restrição das igrejas, comércio, escolas, teatros, cinemas, universidades, transportes público, ou qualquer outro espaço que venha a ocasionar aproximação de pessoas (Oliveira et al., 2020).

Oportunamente, é importante salientar a existência de uma diferenciação entre as definições de distanciamento social, isolamento social e quarentena, definições estas pouco conhecidas, logo, bastante confundidas (Duarte et.al., 2020). $\mathrm{O}$ distanciamento social refere-se à redução do contato entre as pessoas, uma vez existir a possibilidade de pessoas serem infecciosas, mas não identificadas (logo, não estariam em isolamento). $\mathrm{O}$ isolamento, que normalmente ocorre principalmente em hospitais ou em casa, caracteriza-se ao separar uma pessoa acometida de uma doença infecciosa para que esta não transmita às pessoas não doentes. A quarentena, por sua vez, ocorre quando é presumível que uma pessoa se expôs a uma doença contagiosa, embora não apresente sintomas da doença (Wilder-Smith \& Freedman, 2020).

Profilaticamente, a restrição social sob a categoria "distanciamento social”, especificamente, objetiva a prevenção da população tanto a nível individual, como a nível coletivo. Ao longo desse processo, muitas iniciativas de conscientização foram divulgadas no intuito de promover a informação e, consequentemente, a conscientização. Essas medidas objetivam, especialmente, um cuidado com aqueles considerados de alto risco, a exemplo dos idosos, pessoas com comorbidades, portadores de doenças crônicas, tendo em vista a rápida velocidade de agravamento e altos índices de mortalidade (Silva et al., 2020).

Nesse sentido, instituições vêm promovendo diretrizes e propostas para o combate à pandemia: “[...] é fundamental pensar em materiais didáticos de prevenção, em diversas linguagens (pequenos vídeos, panfletos, cartilhas etc.), dirigidas a grupos sociais específicos [...] que leve em conta as diferentes culturas regionais" (CPDH, 2020).

Sob outra ótica, o processo do distanciamento social também tem causado diversos impactos no contexto social, político e econômico. Mudanças quanto ao estilo de vida das pessoas vem sendo bastante apontadas. O estudo realizado por Malta et al. (2020), por exemplo, evidencia que os brasileiros se dedicam menos às atividades físicas, a alimentação está menos saudável em virtude do aumento do consumo de alimentos processados, dedicam maior parte do seu tempo aos computadores e outros tipos de tela, além de estarem consumindo mais álcool e cigarro.

Outra pesquisa, dessa vez desenvolvida por Bezerra et al. (2020), cuja coleta ocorreu em todos os estados brasileiros, avaliou os fatores associados ao comportamento da população durante o isolamento social na pandemia de COVID-19, demonstrando que as percepções individuais acerca do distanciamento social variam de acordo com a renda, idade, escolaridade e sexo.

Para 39\% dos respondentes, o convívio social é o principal aspecto que está sendo afetado pelo isolamento, já $24 \%$ das pessoas relataram o aspecto financeiro como o mais impactante, $19 \%$ dos respondentes informam que o isolamento não está ocasionando nenhum tipo de impacto e $10 \%$ apontam outros fatores que estão sendo impactados, apenas $8 \%$ atribuem o principal impacto à própria saúde. [...] os dados também revelaram que as populações mais pobres já estão sofrendo um impacto maior do isolamento, especialmente em relação à renda (Bezerra et al., 2020, p. 2419).

Certamente, os impactos em diversas esferas são percebidos desde a instauração da pandemia ocasionada pelo SARSCoV-2. A nível econômico, várias repercussões diretas e indiretas em emprego e renda foram observadas. Tanto os trabalhadores informais como os trabalhadores formais foram atingidos, assim como micro e pequenas empresas (Silva \& Silva, 2020).

Segundo a Organização Internacional do Trabalho (OIT, 2020), cerca de 25 milhões de trabalhadores poderão perder seus empregos no curso da pandemia, cujas consequências costumam ser de muita pobreza e vulnerabilidade, especialmente no que tange os países mais emergentes, como o Brasil, quando impactos costumam ser bastante severos. Para tanto, torna-se 
bastante crucial a movimentação e desempenho contundente do governo brasileiro, em todas as suas esferas, no sentido de minimizar os efeitos negativos gerados pela pandemia do COVID-19, cuja crise tende a ser a maior e mais perversa desde a época da segunda guerra mundial.

Mediante esse cenário, o governo brasileiro ampliou as linhas de crédito para micro e pequenos empresários. Contudo, boa parte dos empresários encontraram muitas dificuldades de acesso devido à comprovação de garantia de pagamento. Outras medidas alternativas foram tomadas pelo governo, a exemplo da concessão do auxílio emergencial aos trabalhadores informais, autônomos, microempreendedores individuais e desempregados mediante observação dos critérios estabelecidos. Essas estratégias objetivavam uma minimização dos impactos ocasionados pela pandemia em vários segmentos (Becker, 2020).

Não há dúvidas que grandes desafios serão encontrados na formulação de estratégias sólidas para elaboração e execução de políticas de desenvolvimento econômico para o pós-pandemia que vão muito além da injeção de liquidez da economia e concessão do auxílio emergencial àquelas pessoas mais necessitadas. Não será tarefa simples, uma vez que a crise gerada pela pandemia revelou muitas vulnerabilidades no Sistema Único de Saúde (SUS) e na economia brasileira, a exemplo do número bastante significativo de trabalhadores informais (Costa, 2020).

\subsection{A relação entre covid-19 e saúde mental}

Embora o medo seja um mecanismo de sobrevivência que propicia uma resposta em situações de ameaça, quando desproporcional, gera prejuízos seja pelo aumento de estresse e ansiedade em pessoas saudáveis, seja pela intensificação da sintomatologia de pessoas com transtornos mentais preexistentes (Ornell, 2020a).

$\mathrm{Na}$ existência de doenças infecciosas que geram surtos generalizados, como é o caso do COVID-19, comumente é possível detectar com maior frequência significativos níveis de sofrimento psicológico e maior incidência de sintomas de doenças mentais, embora o impacto à saúde mental leve meses para se tornar efetivamente aparente (Rajkumar, 2020).

Alguns grupos podem se mostrar mais suscetíveis do que outros quanto aos efeitos psicossociais de uma pandemia. É o caso, por exemplo, das pessoas que chegam a contrair a doença, aquelas que se enquadram nos grupos de risco (a exemplo dos idosos, obesos, etc), pessoas com doenças psiquiátricas preexistentes, indivíduos que fazem uso de substâncias, que apresentam funções imunológicas comprometidas, entre outros (Pfefferbaum \& North, 2020). Os grupos especialmente vulneráveis em pandemias costumam, inclusive, sofrer xenofobia, rejeição social e discriminação (Xiang et al, 2020).

Em virtude da alta taxa de mortalidade, medidas mais exigentes foram direcionadas à população idosa. $\mathrm{O}$ distanciamento social que os protegeu do vírus também reduziu sua qualidade de vida. Exemplos de prejuízos que podem vir a desencadear sintomas depressivos na população idosa é a significativa redução das atividades físicas que outrora poderiam ser realizadas ao ar livre, diminuição da procura de suporte à saúde (consultas médicas e exames de rotina), prejuízos no padrão do sono, distorcida compreensão sobre sua saúde, entre outras situações que podem ocasionar maior vulnerabilidade à depressão e sentimentos relativos à solidão (Silva et al, 2020).

Situação semelhante também é possível ser vislumbrada com as crianças. As reações emocionais e alterações do comportamento em crianças se materializam, especialmente, por "dificuldades de concentração, irritabilidade, medo, inquietação, tédio, sensação de solidão, alterações no padrão de sono e alimentação" (Dollabela et al, 2020, s/p).

O distanciamento social presente especialmente na restrição das escolas e suspensão das aulas presenciais vem colaborando para o uso mais frequente dos meios tecnológicos, alterações na rotina de alimentação e padrão do sono das crianças. Assim, comportamentos de estresse e ansiedade começam a se tornar mais evidentes. Logo, boas habilidades parentais são cruciais, especialmente nesse período, pois os pais costumam ser os modelos de comportamento saudável dos seus filhos (Wang et al., 2020). 
Para tanto, no atual contexto é interessante que os pais e cuidadores de uma maneira geral compreendam que se trata não de reações patológicas, mas de reações adaptativas decorrentes de um problema social (Dollabela et al., 2020). Do mesmo modo, é importante que os pais considerem a idade da criança ao conversarem sobre temas relacionados à pandemia, para que possam adaptar a linguagem ao seu entendimento, ou seja, explicando o fenômeno em uma linguagem que seja facilmente compreendida, a fim de gerar mais bem-estar, segurança e conforto emocional da criança (Rodrigues \& Lins, 2020).

Além disso, recomenda-se aos pais uma compreensão mais sensível de que em virtude da grande exposição das crianças às informações, as mesmas podem se encontrar numa fase mais exigente ou até mesmo mais regressiva cabendo aos cuidadores uma postura mais gentil e acolhedora, ensinando-as a expressar seus sentimentos de forma positiva (Ornell et al, 2020a).

Muitos estudos vêm sendo realizados em várias partes do mundo a fim de expor a relação da pandemia gerada pelo COVID-19 e a saúde mental. É válido trazer à baila resultados encontrados em uma revisão sistemática realizada por Vindegaard e Benros (2020) que avaliou as consequências do COVID-19 à saúde mental das pessoas. Os pesquisadores utilizaram como critério de elegibilidade: sintomas psiquiátricos em pacientes com infecção atual por SARS-CoV-2; sintomas psiquiátricos em pacientes psiquiátricos durante a pandemia de COVID-19; e sintomas psiquiátricos/saúde mental de participantes não infectados, divididos em profissionais de saúde e não profissionais da saúde (público em geral).

Seguem os resultados: 96,2\% dos 714 pacientes com COVID-19, hospitalizados, mas estáveis, apresentavam sintomas compatíveis com estresse pós-traumático; $37,5 \%$ de pacientes com transtornos alimentares (transtorno psiquiátrico prévio ao COVID-19) pioraram da sintomatologia do transtorno alimentar durante a pandemia e 56,2\% relatou sintomas de ansiedade (Vindegaard \& Benros, 2020).

Quanto ao público em geral, os estudos indicaram menor bem-estar emocional quando comparado ao momento anterior à pandemia. A maioria dos estudos que compuseram a revisão sistemática indicou o aumento dos sintomas depressivos e ansiogênicos, além da má qualidade do sono presente entre os profissionais de saúde. Níveis mais elevados de sintomas do transtorno obsessivo compulsivo (TOC) foram encontrados em médicos quando comparados a outros profissionais de saúde (Vindegaard \& Benros, 2020).

Profissionais da saúde, em especial os que se encontram na linha de frente do COVID-19, são muito vulneráveis a problemas de saúde mental nesse período. Sentimentos de medo, insegurança pela ausência de equipamentos individuais eficazes, falta de instrução e treinamento adequados são alguns dos fatores que contribuem para esse cenário. As experiências negativas ocasionadas pela prática do trabalho, muitas vezes desassistido, vem ocasionando potenciais sintomas de estresse pós-traumático (Xiang et al, 2020).

Pesquisa realizada por Ramírez-Ortiz et al. (2020) indica que cerca de 10\% dos profissionais de saúde chineses apresentaram sintomas do transtorno de estresse pós-traumático (TEPT), cuja incidência aumentava em até 3 vezes quando familiares ou amigos eram infectados.

Ao passo que o mundo caminhou para uma desaceleração das atividades diárias, com a implementação do distanciamento social, reduzindo as interações entre as pessoas, os profissionais da saúde acabaram trilhando o caminho oposto, pois enfrentaram e ainda enfrentam mais do que nunca grandes jornadas de trabalho, com pouca infraestrutura e defasados equipamentos de proteção gerando desespero, fadiga física e mental, solidão e desamparo (Ornell, 2020b).

Assim, também é crucial a observação da saúde mental dos profissionais da saúde. Os cuidadores também têm a necessidade de serem cuidados. Esse autocuidado para provedores de cuidados da saúde, em especial da saúde mental, pode se dar sob a forma de monitoramento das próprias emoções e reações ao estresse, diluindo o grande número de horas trabalhadas, além da movimentação dos sistemas de saúde no sentido de prover mecanismos de apoio psicossocial quando necessários (Pfefferbaum \& North, 2020). 
Nesse contexto, percebe-se a vulnerabilidade psicológica gerada pela pandemia. Pacientes diagnosticados com COVID-19, indivíduos apenas com a suspeita de COVID-19 ou simplesmente os que se encontram em quarentena ou distanciamento social, profissionais de saúde desassistidos ou grupos considerados de risco tendem a sofrer fortes reações emocionais e comportamentais, como solidão, medo, raiva, tédio, que podem progredir para transtornos depressivos ou de ansiedade, psicóticos, paranoides, inclusive podendo acarretar o suicídio (Ornell, 2020a).

São vários os fatores que podem vir a corroborar com a sintomatologia do estresse e ansiedade. É valido ressaltar que o distanciamento social é um desses fatores, na medida em que limita o direito da população de ir e vir gerando insegurança e incerteza sobre sua duração e eficácia. Por isso, percebe-se certa negação de uma parcela da população do quão grave é essa doença levando, consequentemente, a uma resistência na adesão das medidas de prevenção (Brooks et al., 2020; Enumo et al., 2020 como citado em Pereira et al., 2020).

Um aspecto importante na atuação do profissional de saúde mental, frente às alterações de comportamento em pandemias, diz respeito à abordagem de estilo cognitivos da população em geral. Crenças e distorções cognitivas podem minimizar a adesão às recomendações de profissionais de saúde, diminuindo o efeito das iniciativas governamentais. Nesse sentido, Poland et al. destacam o papel do profissional de saúde na identificação dessas distorções cognitivas (ex.: negação de evidências científicas, crenças baseadas em teorias conspiratórias, generalizações excessivas, heurísticas e vieses) e do uso de intervenções para modificações de tais crenças e comportamentos, por meio de técnicas como a entrevista motivacional e outras (Barros-Delben et al., 2020, p.7)

Para a American Psychiatric Association (APA, 2014), os sintomas ansiogênicos podem ser perceptíveis tanto na sua forma física, como na sua forma psicológica. São exemplos de sintomas físicos: respiração ofegante, palpitações, dores no peito, tremores, enjoos, boca seca, etc. Quanto aos psicológicos, é possível citar: preocupação excessiva, prejuízo na atenção e concentração, nervosismo, medo constante, etc., sintomas esses bastante comuns em outras doenças, inclusive semelhante aos sintomas do próprio COVID-19.

Estratégia bastante utilizada pela Terapia Cognitivo Comportamental (TCC) é a “A.C.A.L.M.E.-S.E.”, composta por 8 passos, quais sejam: 1. Aceite a sua ansiedade; 2. Contemple as coisas em sua volta; 3. Aja com sua ansiedade; 4. Libere o ar de seus pulmões; 5. Mantenha os passos anteriores; 6. Examine seus pensamentos; 7. Sorria, você conseguiu; 8. Espere o futuro com a aceitação (Rangé \& Borba, 2008 como citado em Rolim; Oliveira \& Batista, 2020).

Em virtude da mudança brusca e repentina do estilo de vida, os sintomas de ansiedade podem, inclusive, evoluir para um quadro depressivo. Pesquisa realizada por Barros et al. (2020) aponta que o sentimento relacionado à tristeza ou até mesmo à depressão atingiu em torno de $40 \%$ dos adultos brasileiros, ao passo que a ansiedade e nervosismo estiveram presentes em mais de 50\%, cuja prevalência foi mais significativa em adultos jovens, pessoas do sexo feminino ou com diagnóstico anterior de depressão.

O sofrimento mental pode corroborar inclusive no surgimento do TEPT quando o indivíduo fica suscetível a pensamentos negativistas e suicidas incompatíveis com a visão positiva que possuía antes do trauma. Além de um ambiente seguro e confiável, são condutas dentro do protocolo proposto pela TCC aos indivíduos com TEPT:

Psicoeducação sobre estresse traumático e TCC; antecipar e superar o comportamento de esquiva; automonitorar a ligação entre eventos, pensamentos e emoções; identificar crenças sobre o motivo do evento traumático e suas consequências; uso de diálogo socrático em relação a avaliação que o paciente faz sobre o trauma; estratégias para desafiar crenças e pensamentos sobre o trauma e suas consequências; após o término acompanhamento para avaliar manutenção dos ganhos e necessidade de mais terapia (Monson \& Shnaider, 2014 como citado em Peuker et al, 2020, p.15).

Ainda, é válido destacar que o sofrimento ocasionado pela pandemia vem sendo reportado como indicativo de suicídio, seja pelo aumento do medo, da preocupação, do desemprego, do uso de álcool e outras drogas, entre outros. Revisão 
sistemática incluindo quatorze artigos indica que 4 deles alertam o aumento da taxa de suicídio ao longo da pandemia. Alguns deles reportam ainda que o distanciamento social, a crise econômica, responsável pelo desemprego em massa, ou até mesmo a discriminação com os infectados são potenciais fatores de aumento da taxa de tentativa de suicídio (Schuck, 2020).

Estratégias de enfrentamento, intervenções e criação de manejos que abarquem a dimensão social e psicológica dos indivíduos passou a ser fundamental para fortalecer a população para tornar esse período o menos doentio possível. Algumas questões poderiam ser incrementadas com mais afinco, a exemplo de equipes multidisciplinares e comunicações claras e precisas à população sobre o COVID-19. Compreendendo o estado de saúde mental das diferentes populações, identificando as pessoas que se encontram em alto risco de suicídio e agressão e fornecendo serviços psicológicos aos que precisam são passos importantes no cuidado à saúde mental em tempos de pandemia (Ramírez-Ortiz, 2020).

Desse modo, práticas e políticas públicas precisam ser elaboradas e postas em prática. Sugere-se que as intervenções sejam formuladas com base no grau de risco. A OMS as classifica em Universais: quando as intervenções são voltadas a toda população, objetivando impedir o surgimento de qualquer comportamento alvo; Seletivas: quando as intervenções se concentram em pessoas e populações que se encontram em baixo risco através do acolhimento e informação; e Indicadas, que são intervenções direcionadas às pessoas com risco iminente ou que já apresentaram um comportamento alvo (Greff, 2020).

O Conselho Federal de Psicologia (CFP, 2020), por intermédio da Resolução $n^{\circ}$ 04/2020, orienta os psicólogos à atuação na modalidade online diante da pandemia do COVID-19. Essa ferramenta vem sendo bastante útil, especialmente por trazer mais flexibilidade aos psicólogos no atendimento remoto diante da dificuldade dos atendimentos presenciais durante períodos de distanciamento social.

Estima-se que um terço ou até mesmo a metade da população mundial venha a apresentar algum transtorno mental ao longo da pandemia (FIOCRUZ, 2020). Logo, para que não se instaure uma "pandemia" relativa à saúde mental, com alta incidência de distúrbios depressivos e de ansiedade, algumas atitudes podem fazer a diferença, por exemplo: dormir bem, realizar atividades físicas, equilibrar a dieta, meditar e praticar exercícios de relaxamento ou outras medidas que fortaleçam um estilo de vida saudável. Logo, estratégias de prevenção são relevantes, pois uma saúde mental prejudicada reflete diretamente numa baixa expectativa de vida (Raony et al, 2020).

Segundo a abordagem da TCC, através da psicoeducação, é possível ensinar ao paciente formas de lidar com as doenças, sejam elas físicas ou mentais. Além disso, ainda dentro dessa abordagem, é possível ensinar técnicas de respiração e relaxamento para que o paciente possa regular melhor sintomas ansiogênicos ou depressivos (Pereira et al, 2020).

Tendo em vista a significativa demanda de pessoas em busca de ajuda profissional na área da saúde mental atualmente, percebe-se a importância da profissão do psicólogo, uma vez que ele auxiliará seus clientes na regulação de suas emoções, na superação do sofrimento e crises ocasionados por um período tão perturbador (Pimentel et al, 2020). Aos colaboradores que tiveram sua modalidade de trabalho transformada em teletrabalho, sem que houvesse preparo para essa transição, (Chong; Huang \& Chang, 2020) é também muito importante a atuação do psicólogo atuante nas organizações.

Por derradeiro, vale lembrar que um momento de crise não somente se perfaz por seus aspectos negativos (distanciamento social, redução da autonomia, iminência do adoecimento), mas também uma oportunidade de transformação daqueles que a vivenciam, de interpretação de que a crise também pode ser vista como um momento de novas possibilidades diante de mudanças auto impostas, a exemplo da realização de atividades cotidianas ou laborais em outros formatos representando, assim, um importante divisor de águas no contexto de organização social (Barros-Delben et al, 2020).

\section{Considerações Finais}

O ser humano está em constante mudança e evolução, já foi capaz de adaptar-se a diversas situações extremas, mas os impactos causados pela COVID-19 afetaram o âmbito social, emocional, ambiental, organizacional, entre outros. Dessa forma, 
partir desse trabalho, atentou-se aos desafios impostos à saúde mental diante da pandemia por COVID-19, destacando-se o distanciamento social, fator de mudança relevante na sociedade, principalmente em uma cultura que preza pelo toque e pelo contato físico como demonstração de afeto e educação, além do medo diante de um vírus que poderia ser letal para qualquer pessoa.

Assim, à guisa de conclusão, a literatura indica que boa parte da população foi afetada, desenvolvendo algum transtorno mental nesse período, com destaque para a ansiedade e a depressão. Portanto, faz-se imprescindível a criação de estratégias para promover qualidade de vida e bem-estar em tempos tão propícios para o adoecimento mental, o que torna essencial a atuação do psicólogo nesse contexto. É esperada a expansão da literatura sobre os impactos causados pela COVID19 em todos os âmbitos possíveis, inclusive quanto à saúde mental, que por um bom tempo ainda será afetada ainda que indiretamente por esse fenômeno adverso, de modo a se reparar, mensurar e cuidar dos danos gerados pela pandemia.

Por derradeiro, levando-se em consideração certa carência de pesquisas acerca da temática, especialmente com altos níveis de evidência, assim como a subsistência de desafios que ainda precisam ser enfrentados e superados, entende-se pela relevância da realização de estudos mais amplos, mediante um olhar mais amplo e sensível lançado à realidade, que possam mensurar e detalhar as repercussões do Covid-19, de modo a embasar ações e planos de iniciativa individual e plural que valorizem o cuidado, prevenção e promoção da saúde mental em tempos de Covid-19, a fim de minimizar os impactos avassaladores decorrentes da atual pandemia. É importante a criação de estratégias que promovam a qualidade de vida e o bem-estar em momentos tão favoráveis ao adoecimento mental.

\section{Referências}

American Psychiatric Association (2014). Manual Diagnóstico e Estatístico de Transtornos Mentais: DSM-5. (5a ed.), Artmed.

Barros-Delben, P. et al. (2020). Saúde mental em situação de emergência: Covid-19. Debates em Psiquiatria (Associação Brasileira de Psiquiatria), 10(2), 1828.

Barros, M. B. A. et al. (2020). Relato de tristeza/depressão, nervosismo/ansiedade e problemas de sono na população adulta brasileira durante a pandemia de COVID-19. Epidemiol. Serv. Saúde, 29(4), 1-12.

Becker, K. L. (2020). Ampliação do Programa Benefício de Prestação Continuada (Bpc): Essencial Para Amenizar A Pobreza e Urgente em Tempos de Pandemia.

Bezerra, A. C. V., Silva, C. E. M., Soares, F. R. G, \& Silva, J. A. M. (2020). Fatores associados ao comportamento da população durante o isolamento social na pandemia de COVID-19. Ciênc. saúde coletiva, 25(1), 2411-2421.

Biblioteca virtual de saúde - BVS (2021). Saúde mental e a pandemia de Covid-19. Ministério da Saúde.

Brasil. (2021a). Ministério da Saúde. [Internet]. https://bit.ly/3o6HG9d.

Brasil. (2021b). Ministério da Saúde. [Internet]. https://bit.ly/3bjXfVO.

Brasil. (2021c). Ministério da Saúde. [Internet]. https://bit.ly/3ffW6Qp.

Cavalcante, J. R. et al. (2020). COVID-19 no Brasil: evolução da epidemia até a semana epidemiológica 20 de 2020. Epidemiol. Serv. Saúde, 29 (4), 1-13.

Chong, S., Huang, Y., \& Chang, C. (2020). Supporting interdependent telework employees: A moderated-mediation model linking daily COVID-19 task setbacks to next-day work withdrawal. Journal of Applied Psychology, 105(12), 1408-1422.

Conselho Federal de Psicologia. (2020). Resolução CFP número 04 de 26 de março de 2020. Dispõe sobre regulamentação de serviços psicológicos prestados por meio de Tecnologia da Informação e da Comunicação durante a pandemia do COVID-19.

Costa, S. S. (2020). Pandemia e desemprego no Brasil. Rev. Administração Pública, 54(4), 969-978.

CPDH. ABONG, ARL, CMP (2020). Covid 19 nas periferias urbanas, favelas e grupos sociais vulneráveis. Proposta de estratégias de ação.

Dollabella, B. M. et al. (2020). Saúde mental e atenção psicossocial na pandemia COVID-19: crianças na pandemia Covid-19. Fiocruz/CEPEDES, 20 p. Cartilha.

Domingues, C. M. A. S. (2021). Desafios para a realização da campanha de vacinação contra a COVID-19 no Brasil. Cad. Saúde Pública, 37 (1), 1-5.

Duarte, M. Q., Santo, M. A. S., Lima, C. P., Giordani, J. P., \& Trentini, C. M. (2020). COVID-19 e os impactos na saúde mental: uma amostra do Rio Grande do Sul, Brasil. Ciênc. saúde coletiva, 25(9), 3401-3411. 
Fiocruz, Fundação Oswaldo Cruz (2020). Cartilha Saúde Mental e Atenção Psicossocial - Recomendações para Gestores.

Greff, A. P. et al. (2020). Saúde mental e atenção psicossocial na pandemia COVID-19: suicídio na pandemia COVID-19. Fiocruz, 24 p. Cartilha.

Guan, W. et al. (2019). Clinical Characteristics of Coronavirus Disease 2019 in China. New England Journal of Medicine.

Iser, B. P. M., Silva, I., Raymundo, V. T., Poleto, M. B., Schuelter-Trevisol, f., \& Bobinski, F. (2020). Definição de caso suspeito da COVID-19: uma revisão narrativa dos sinais e sintomas mais frequentes entre os casos confirmados. Epidemiol. Serv. Saúde, 29(3), 1-11.

Laville, C., \& Dionne, J. (1999). A construção do saber: manual de metodologia da pesquisa em ciências humanas. Porto Alegre: Artes Médicas Sul; Belo Horizonte: UFMG.

Malta, D. C. et al. (2020). A pandemia da COVID-19 e as mudanças no estilo de vida dos brasileiros adultos: um estudo transversal. Epidemiol Serv Saúde, $29(4), 1-13$.

Mao, L. et al. (2020). Neurological manifestations of hospitalized patients with COVID-19 in Wuhan, China: a retrospective case series study. AMA Neurol, $1-$ 26.

Marconi, M. A.; \& Lakatos, E. M. Metodologia do Trabalho Científico. (8a ed.), Atlas.

Noronha, K. V. M. S. et al. (2020). Pandemia por COVID-19 no Brasil: análise da demanda e da oferta de leitos hospitalares e equipamentos de ventilação assistida segundo diferentes cenários. Cad. Saúde Pública, 36(6), 1-17.

Oliveira, W. K., Duarte, E., França, G. V. A., Garcia, L. P. et al. (2020). Como o Brasil pode deter a COVID-19. Epidemiol. Serv. Saúde, 29(2), 1-8.

Ornell, F., Schuch, J. B., Sordi, A. O., \& Kessler, F. H. P. (2020a). "Pandemic fear" and COVID-19: mental health burden and strategies. Braz. J. Psychiatry, 42(3), 232-235.

Ornell, F., Halpern, S. C., Kessler, F. H. P., \& Narvaez, J. C. M. (2020b). Cad. Saúde Pública, 36(4), 1-6.

Pereira, M. D. et al. (2020). The COVID-19 pandemic, social isolation, consequences on mental health and coping strategies: an integrative review. Research, Society and Development, 9(7), 1-31.

Peuker, A. C., Almondes, K. M., Miyazaki, M. C. O. S. \& Soares, M. R. Z. (2020). Manejo do estresse em níveis traumáticos em trabalhadores e pacientes de COVID-19. Saúde mental e COVID 19.

Pfefferbaum, B., \& North, C.S. (2020). Mental Health and the Covid-19 Pandemic. N Engl J Med, 383(6), 510-512.

Pimentel, A. S. G., Maués, H. P., Lima, N. C. F. de., \& Andrade Jr. G. F. (2020). Orientações da Psicologia brasileira em relação a prevenção da Covid19. Rev. NUFEN, 12(2), 102-117.

Rajkumar, R. P. (2020). COVID-19 and mental health: A review of the existing literature. Asian Journal of Psychiatry, 52, 1-5.

Ramírez-Ortiz, J., Castro-Quintero, D., Lérma-Córdoba, C., Yéla-Ceballos, F., Escobar-Córdoba, F. (2020). Consecuencias de la pandemia COVID-19 en la Salud Mental asociadas al aislamiento social. SciELO Preprints, 1-21.

Raony, Í., Figueiredo, C. S., Pandolfo, P., Araújo, E. G., Bomfim, P. O., \& Savino, W. (2020). Psycho-Neuroendocrine-Immune Interactions in COVID-19: Potential Impacts on Mental Health. Frontiers in Immunology, 11, 1-15.

Rodrigues, J. V. S., \& Lins, A. C. A. A. Possíveis impactos causados pela pandemia da COVID-19 na saúde mental de crianças e o papel dos pais neste cenário. Research, Society and Development, 9(8), 1-9.

Rolim, J. A., Oliveira, A. R., \& Batista, E. C. (2020). Manejo da ansiedade e COVID-19. Rev Enfermagem e Saúde Coletiva, 4(2), 64-74.

Schuck, F. W., Weber, G. M. F., Schaefer, C. K., Renheimer, M. W., \& Rockenbach, D. M. (2020). A influência da pandemia de COVID-19 no risco de suicídio. Braz. J. Hea. Rev., 3(5), 13778-13789.

Sifuentes-Rodríguez, E., \& Palacios-Reyes, D. (2020). Covid-19: The outbreak caused by a new coronavirus. Bol Med Hosp Infant Mex, 77(2), 47-53.

Silva, M. V. S., Rodrigues, J. A., Ribas, M. S., Sousa, J. C. S, Castro, T. R. O., Santos, B. A., Sampaio, J. M. C, \& Pegoraro, V. A. (2020). O impacto do isolamento social na qualidade de vida dos idosos durante a pandemia por COVID-19. Enferm Bras., 19(4), 34-41.

Silva, M. L., \& Silva, R. A. (2020). Economia brasileira pré, durante e pós-pandemia do covid-19: impactos e reflexões. UFSM, FAPERGS.

Vindegaard, N., \& Benros, M. E. (2020). COVID-19 pandemic and mental health consequences: systematic review of the current evidence. Brain, Behavior, and Immunity, 89, 531-542.

Wang, Z., Yang, B., Li, Q., Wen, L., \& Zhang, R. (2020). Clinical features of 69 cases with coronavirus disease 2019 in Wuhan, China. Clin Infect Dis., 71(15), 769-777.

Wilder-Smith, A., \& Freedman. D, O. (2020). Isolation, quarantine, social distancing and community containment: pivotal role for old-style public health measures in the novel coronavirus (2019-nCoV) outbreak. J Travel Med, 27(2), 1-4.

World Health Organization - Who. (2021). Coronavirus disease (COVID-19) pandemic. World Health Organization.

Xiang, Y. T., Yang, Y., Li, W., Zhang, L., Zhang, Q., Cheung, T., et al. (2020). Timely mental health care for the 2019 novel coronavirus outbreak is urgently needed. Lancet Psychiatry, 7(3), 228-229. 